\title{
Similarity Measurement Between Images
}

\author{
Chaur-Chin Chen *and Hsueh-Ting Chu \\ Department of Computer Science \\ National Tsing Hua University \\ Hsinchu 300, Taiwan \\ E-mail: cchen@cs.nthu.edu.tw
}

\begin{abstract}
Experimental results of applying two similarity measurements, Euclidean distance and chord distance, to test a set of six Brodatz's textures are reported. Experiments show that in addition to feature extraction, A similarity measurement between images should be simultaneously considered, We also review some other similarity measurements.
\end{abstract}

\section{Introduction}

In a digital multimedia era, the research of contentbased image retrieval (CBIR) used to establish a database composed of images, each is represented as a vector of features derived from color, shape, and/or texture information. When the query is requested, a similarity measurement between a user-provided image and those prestored in the database is computed and compared to report a few of most similar images. In the process, texture features from Gabor, Daub4, and Haar [3, 5] were commonly extracted as representative vectors for CBIR although no universally best set of texture features ever exists. Most of the existing works highlight a high matched retrieval rate in author-specified databases by using conventional classifiers such as 1-nn classifier, Bayesian classifier, or a Fisher's discriminant $[2,4]$. We have realized that the recognition rate in a pattern recognition system should simultaneously consider feature extraction and classifier design [2]. However, a similarity measurement between two images was seldom investigated. This paper studies the effect of similarity measurement between texture features derived from Gabor and wavelet transforms Daub4 and Haar. We report experimental results on a database of Brodatz's textures [1].

*Chaur-Chin Chen was partially supported by NSC Grant 93-2213-E-007-053

\section{Similarity Measurement}

A similarity measurement must be selected to decide how close a vector is to another vector. The problem can be converted to computing the discrepancy between two vectors $\mathbf{x}, \mathbf{y} \in R^{d}$. This paper examines the three distance measurements: Euclidean, Mahalanobis, and chord distances which are reviewed as follows.

\subsection{Euclidean Distance}

The Euclidean distance between $\mathbf{x}, \mathbf{y} \in R^{d}$ is computed by

$$
\delta_{1}(\mathbf{x}, \mathbf{y})=\|\mathbf{x}-\mathbf{y}\|_{2}=\sqrt{\sum_{j=1}^{d}\left(x_{j}-y_{j}\right)^{2}}
$$

A similar measurement called the cityblock distance, which takes fewer operations, is computed by

$$
\tau_{1}(\mathbf{x}, \mathbf{y})=\|\mathbf{x}-\mathbf{y}\|_{1}=\sum_{j=1}^{d}\left|x_{j}-y_{j}\right|
$$

Another distance measurement called the supreme norm, is computed by

$$
\tau_{2}(\mathbf{x}, \mathbf{y})=\max _{1 \leq j \leq d}\left|x_{j}-y_{j}\right|
$$

\subsection{Mahalanobis Distance}

The Mahalanobis distance between two vectors $\mathbf{x}$ and $\mathbf{y}$ with respect to the training patterns $\left\{\mathbf{x}_{i}\right\}$ is computed by

$$
\delta_{2}(\mathbf{x}, \mathbf{y})=\sqrt{(\mathbf{x}-\mathbf{y})^{t} S^{-1}(\mathbf{x}-\mathbf{y})}
$$

where the mean vector $\mathbf{u}$ and the sample covariance matrix $S$ from the sample $\left\{\mathbf{x}_{i} \mid 1 \leq i \leq n\right\}$ of size $n$ are computed by $S=\frac{1}{n} \sum_{i=1}^{n}\left(\mathbf{x}_{i}-\mathbf{u}\right)\left(\mathbf{x}_{i}-\mathbf{u}\right)^{t}$ with $\mathbf{u}=\frac{1}{n} \sum_{i=1}^{n} \mathbf{x}_{i}$ 


\subsection{Chord Distance}

The chord distance between two vectors $\mathbf{x}$ and $\mathbf{y}$ is to measure the distance between the projected vectors of $\mathbf{x}$ and $\mathbf{y}$ onto the unit sphere, which can be computed by

$$
\delta_{3}(\mathbf{x}, \mathbf{y})=\left\|\frac{\mathbf{x}}{r}-\frac{\mathbf{y}}{s}\right\|_{2},
$$

where $r=\|\mathbf{x}\|_{2}, s=\|\mathbf{y}\|_{2}$. A simple computation leads to $\delta_{3}(\mathbf{x}, \mathbf{y})=2 \sin (\alpha / 2)$ with $\alpha$ being the angle between vectors $\mathbf{x}$ and $\mathbf{y}$. The smaller the angle $\alpha$, the closer the two vectors $\mathbf{x}$ and $\mathbf{y}$.

A similar measurement based on the angle between vectors $\mathbf{x}$ and $\mathbf{y}$ is defined as

$$
\tau_{3}(\mathbf{x}, \mathbf{y})=1-|\cos (\alpha)|, \quad \cos (\alpha)=\frac{\mathbf{x} \cdot \mathbf{y}}{\|\mathbf{x}\|_{2}\|\mathbf{y}\|_{2}}
$$

\subsection{Pearson's Correlation Coefficient}

A measurement derived from Pearson correlation coefficient $\rho(\mathbf{x}, \mathbf{y})$ is defined as

$\delta_{4}(\mathbf{x}, \mathbf{y})=1-|\rho|, \quad \rho=\frac{\sum_{i=1}^{d}\left(x_{i}-u\right)\left(y_{i}-v\right)}{\left(\sqrt{\left[\sum_{i=1}^{d}\left(x_{i}-u\right)^{2}\right]} \sqrt{\left[\sum_{i=1}^{d}\left(y_{i}-v\right)^{2}\right]}\right.}$

The larger $|\rho|$ is, the closer the vectors $\mathbf{x}$ and $\mathbf{y}$ are.

\subsection{Spearman Rank Coefficient}

A measurement derived from Spearman rank coefficient $\gamma(\mathbf{x}, \mathbf{y})$ is defined as

$$
\delta_{5}(\mathbf{x}, \mathbf{y})=1-\frac{6 \sum_{j=1}^{d} r_{j}^{2}}{d\left(d^{2}-1\right)}
$$

where $r_{j}=x_{(j)}-y_{(j)}$ is the rank difference between the components of vectors $\mathbf{x}$ and $\mathbf{y}$. Note that $-1 \leq \delta_{5} \leq 1$. the larger $\delta_{5}$ is, the closer the vectors $\mathbf{x}$ and $\mathbf{y}$ are.

\section{$3 \quad$ Experimental Results}

Texture features computed from Gabor, Daubechies' four (Daub4) and Haar transforms [3] tested on 96 textures with 6 categories are given in Table 1 . The texture images are shown in Figure 1.

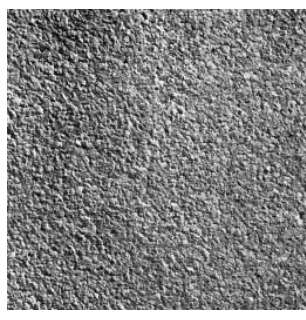

(a) pressed cork

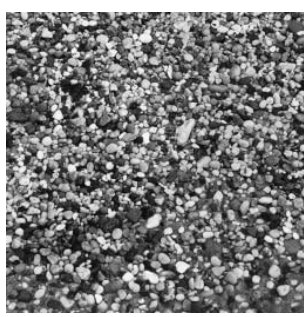

(d) beach pebbles

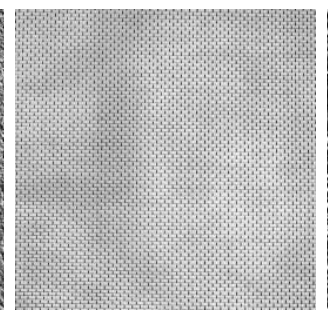

(b) aluminum wire

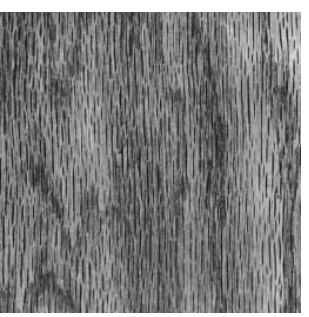

(e) wood grain

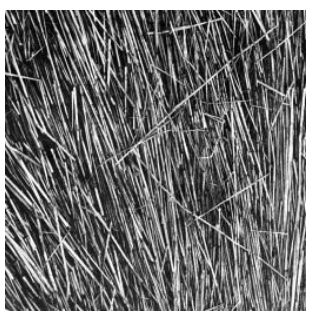

(c) straw

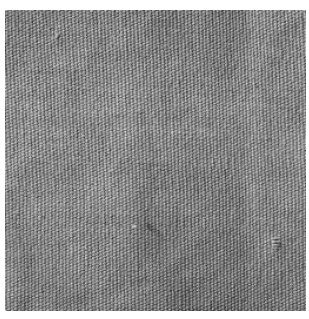

(f) cotton canvas
Figure 1: Six Brodatz's Textures: (a) D04, (b) D06, (c) D15, (d) D54, (e) D68, (f) D77.

Table 2: Leave-one-out Errors on Six Brodatz's

\begin{tabular}{|cccc|}
\hline \multicolumn{4}{c}{ Textures. } \\
\hline$\delta_{j}$ & Gabor & Daub4 & Haar \\
\hline Euclidean & $4 / 96$ & $0 / 96$ & $0 / 96$ \\
\hline Chord & $8 / 96$ & $0 / 96$ & $3 / 96$ \\
\hline
\end{tabular}

\section{References}

[1] P. Brodatz, Textures - A Photographic Album for Artists and Designers, Dover, New York, 1966.

[2] C.C. Chen, C.C. Chen, Texture Synthesis: A Review and Experiments, Journal of Information Science and Engineering 19, 371-380, 2003.

[3] I. Daubechies: Ten Lectures on Wavelets, Society for Industrial and Applied Mathematics, Philadelphia 1992.

[4] J. Peng, D.R. Heisterkamp, H.K. Dai, Adaptive quasiconformal kernel nearest neighbor classification, IEEE Trans. Pattern Anal. Machine Intell. 26, 656-661, 2004.

[5] J. Zhang, T. Tan, Brief review of invariant texture analysis methods, Pattern Recognition 35, 735-747, 2002. 